\title{
Deep Features and Data Reduction for Classification of SD-OCT Images: Application to Diabetic Macular Edema
}

\author{
Genevieve C. Y. Chan, Syed A. A. Shah, T. B. Tang, C.-K. Lu, H. Muller* and F. Meriaudeau \\ Centre for Intelligent Signal and Imaging Research Department of Electrical and Electronic \\ Engineering, Universiti Teknologi PETRONAS \\ 32610 Bandar Seri Iskandar, Perak Darul Ridzuan, Malaysia. \\ genevieve.chan94@gmail.com,fabrice.meriaudeau@utp.edu.my
}

\author{
*University of Applied Sciences Western Switzerland, Sierre (HES-SO) Rue du TechnoPôle \\ 3, 3960 Sierre, Switzerland
}

\begin{abstract}
Diabetic Macular Edema (DME) is defined as the accumulation of extracellular fluids in the macular region of the eye, caused by Diabetic Retinopathy (DR) that will lead to irreversible vision loss if left untreated. This paper presents the use of a pre-trained Convolutional Neural Network (CNN) based model for the classification of Spectral Domain Optical Coherence Tomography (SD- OCT) images of Diabetic Macular Edema (DME) with feature reduction using Principal Component Analysis (PCA) and Bag of Words (BoW). The model is trained using SD-OCT dataset retrieved from the Singapore Eye Research Institute (SERI) and is evaluated using an 8-fold cross validation at the slide level and two patient leave out at the volume level. For the volume level, an accuracy of $96.88 \%$ is obtained for data that was preprocessed.
\end{abstract}

Index Terms - CNNs, Dimension reduction, SDOCT, Diabetic Macular Edema

\section{INTRODUCTION}

The Macula of the eye is the part of the retina that controls our most detailed vision. Diabetic Macular Edema (DME) is defined as an abnormal collection of extracellular fluid that increases the retinal thickness due to leakage of blood vessels in the retina caused by the Diabetic Retinopathy (DR) - a disease that damages the blood vessels in the retina [1]. It is a leading cause of irreversible blindness among diabetic patients, especially Type II diabetes if DME is left untreated, A report from the Obesity Prevention Council president, Jong Koi Chong states that in 2006, 8.6\% of the working- Malaysians had diabetes mellitus and within 5 years, it increased to $15.2 \%$ and the number is still expected to rise. In 2013, Malaysia has become the country in Asia having the highest number of diabetic patients with a total of 3.6 million [2].

Fundus photography has been the most common tool for assessing the retina or macula; indeed, fundus images can easily reveal micro-aneurisms [3], exudates [4] that are the most common signs among patients suffering from
DR. On the other hand, DME signs are harder to visualize through a $2 \mathrm{D}$ projection of the retina, as the swelling is set to diminish with the perspective effect and ophthalmologists prefer to use Spectral Domain Optical Coherence Tomography (SD-OCT) [5]. SDOCT is able to probe through the retina depth and can image the retinal layers at a higher resolution and thus providing better quality imaging for diagnosis [6]. While the imaging part is still improving, the Computer Aided Diagnosis community started to embrace deep learning architectures especially Convolutional Neural Networks (CNNs) [7, 8]. Recently, Burlina et al. [9] used transfer learning to classify patients with Age-related Macular Degeneration (AMD) while Karri et al. [10] use GoogLeNet on both DME and AMD SD-OCT data. In the first case, the features extracted with Overfeat [11] fed to a linear SVM led to an accuracy between $92 \%$ and $95 \%$ whilst for the second case, the authors obtained an accuracy of $96 \%$ with a linear SVM classifier. Both datasets undergo image preprocessing: Burlina et al. - cropping and segmentation; Karri et al. - BM3D filtering, retinal flattening cropping and image pyramid construction.

Prior to recent deep architectures, a review was conducted to compare state of the art research methods on classifying DME and normal patients using SDOCT volumes [12]. The survey reviewed six recent papers: Venhuizen et al. [13], Srinivasan et al. [14], Lemaitre et al. [15], Alsaih et al. [16], Liu et al. [17] and Sankar et al. [18]. It is highlighted that all researchers except Srinivasan et al. use PCA for feature reduction while Veinhuizen et al., Lemaitre et al. and Liu et al. use BoW to reduce the number of dimensions. All approaches go through preprocessing, feature extraction, mapping, feature reduction and finally classification. The research with the highest sensitivity (Lemaitre et al. [15]) of $87.5 \%$ undergoes image denoising using Non Local Means (NLM), extracts Texton features, PCA and BoW feature reduction and finally classifies using Random Forests (RF). The approach with the highest specificity (Liu et al. [17]) of $93.8 \%$ undergoes image flattening and alignment, extracting the edges uses Local Binary Patterns 
(LBP) for computation, PCA and BoW for feature space reduction and finally an SVM as the classifier.

More recently, on the same dataset, Chan et al. [19] introduced deep learning through transfer learning using the AlexNet model [20], evaluated within slice level and achieved a very high accuracy of $96 \%$. On the other hand, Awais et al. [21] use VGGNet to classify the SD-OCT images within volume level using the same dataset and has achieved $87 \%$ accuracy. Extending the previous work [19], this paper further investigates the effect of deep features associated with feature reduction using Principal Component Analysis (PCA) and Bag of Words $(\mathrm{BoW})$ on image classification performance.

The remainder of this paper is organized as follows: In Section II we present the methodology of the proposed algorithm. Section III shows the results and discusses the results. The paper is concluded in section IV.

\section{METHODOLGY}

The proposed system consists of two preprocessing steps detailed in [19]: noise removal with BM3D (Fig. 1) and image size reduction via cropping (after having detected the retina layers, see Fig 2), feature extraction, feature space reduction and classification.

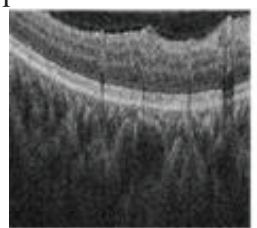

(a)

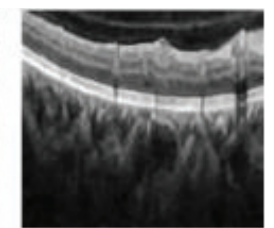

(b)
Fig 1. BM3D Filtering, (a) noisy images, (b) BM3D Filtered

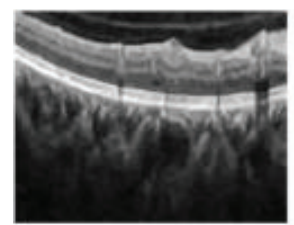

(a)

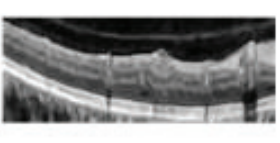

(b)
Fig 2. BM3D Filtering, (a) BM3D Filtered, (b) Cropped image once retinal layers are detected

\subsection{Features extraction}

The data set used is from the Singapore Eye Research Institute (SERI) [15]. It consists of $32 \mathrm{SD}-\mathrm{OCT}$ volumes including 16 normal and 16 DME volumes with $128 \times 1024 \times 512$. The images are resized to $227 \times 227 \times 3$ before being presented to AlexNet for feature extraction. The architecture of AlexNet [20], consists of five convolutional layers and three fully connected layers (FC). The Features are extracted at the three last FCN layers (FC6, FC7 and FC8).

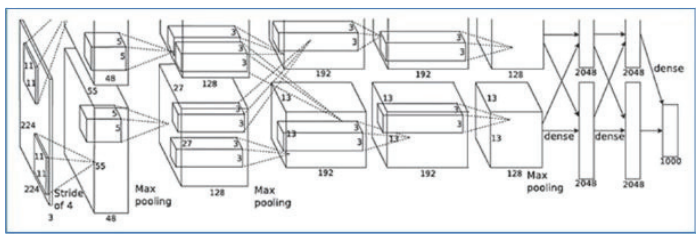

Fig. 3 Illustration of the AlexNet Architecture using a CNN along with the filter bans value at each layer. Adapted from "ImageNet Classification with Deep Convolutional Neural Networks" by Krizhevsky A. et al. (2012) [20].

\subsection{Feature space reduction}

For feature space reduction PCA [22] and BoW are investigated [23]. For PCA, 60 to 110 eigenvalues, representing 85 to $99 \%$ of the signal energy, depending on the feature vector size FC6 (4096), FC7 (4096) or FC8 (1000) were tested while varying the preprocessing (denoising or not, cropping or not). The dictionary size was also changed from 60 to 110 for the BoW.

\subsection{Training and testing}

Two strategies were investigated. In both cases a linear SVM was used as the classifier. For the classification of SD-OCT images, an 8-fold crossvalidation was used. For volume classification, the leave two out strategy was used; i.e. out of 32 volumes, 30 volumes are used in training while the remaining 2 volumes (one DME one normal) are used in testing; the final score being taken as a majority vote.

\section{RESULTS}

Nearly 500 experiments were run with varying parameters. The table below only reports some of the best performing approaches. Overall, PCA associated with deep features shows very good performance with sensitivity of $93.75 \%$ specificity of $100.00 \%$ and accuracy of $96.8 \%$ at the volume level for the best performance. These results outperform the previously published results on the same dataset by Awais et al. [21] which reported an accuracy of $87 \%$ but with a lower sensitivity (between $75 \%$ and $87 \%$ depending on the configuration) and comfort the fact that preprocessing and data reduction are useful in the case of noisy images and a sparse feature space. At the image level, the highest performance is with a sensitivity of $84.03 \%$, a specificity of $89.26 \%$ and an accuracy of $86.65 \%$ which is slightly lower than previous reported results [19] where the authors used a linear SVM in a high dimensional feature space with few data points filling the space and subject to a questioning about generalization. Overall, BoWs showed poor performance and a maximum accuracy of only $81.25 \%$ at the volume level for non-processed images while at the same time, the classification at the image level was very poor, highlighting the fact that some randomness still exists after the BoW. 
Table 1: summary of the best results obtained for PCA data reduction

\begin{tabular}{|c|c|c|c|c|c|c|c|c|c|c|}
\hline \multicolumn{2}{|c|}{ Pre-processing } & \multirow{3}{*}{$\begin{array}{c}\text { Fully } \\
\text { Connected } \\
\text { Layer }\end{array}$} & \multirow{3}{*}{$\begin{array}{l}\text { Feature } \\
\text { Size }\end{array}$} & \multirow{3}{*}{$\begin{array}{c}\text { Energy } \\
(\%)\end{array}$} & \multicolumn{6}{|c|}{ Evaluation (\%) } \\
\hline \multirow{2}{*}{$\begin{array}{l}\text { Noise } \\
\text { Removal }\end{array}$} & \multirow{2}{*}{$\begin{array}{l}\text { Image } \\
\text { Cropping }\end{array}$} & & & & & Slice & & & Volume & \\
\hline & & & & & SE & SP & $\mathrm{ACC}$ & SE & SP & $\mathrm{ACC}$ \\
\hline \multirow{8}{*}{ No } & \multirow{8}{*}{ No } & \multirow{3}{*}{ FC6 } & 60 & 91.31 & 69.34 & 81.01 & 75.17 & 87.50 & 100.00 & 93.75 \\
\hline & & & 70 & 92.32 & 70.07 & 81.54 & 75.81 & 87.50 & 100.00 & 93.75 \\
\hline & & & 80 & 93.13 & 69.92 & 80.96 & 75.44 & 87.50 & 93.75 & 90.63 \\
\hline & & \multirow{3}{*}{$\mathrm{FC7}$} & 90 & 96.56 & 68.36 & 80.03 & 74.19 & 87.50 & 100.00 & 93.75 \\
\hline & & & 100 & 96.94 & 69.58 & 80.66 & 75.12 & 87.50 & 100.00 & 93.75 \\
\hline & & & 110 & 97.25 & 70.46 & 81.74 & 76.10 & 81.25 & 100.00 & 92.71 \\
\hline & & \multirow{2}{*}{ FC8 } & 100 & 99.20 & 70.95 & 78.56 & 74.76 & 81.25 & 93.75 & 87.50 \\
\hline & & & 110 & 99.31 & 69.19 & 78.22 & 73.71 & 81.25 & 93.75 & 87.50 \\
\hline \multirow{7}{*}{ Yes } & \multirow{7}{*}{ No } & \multirow{3}{*}{ FC6 } & 90 & 92.24 & 72.71 & 86.08 & 79.39 & 81.25 & 100.00 & 90.63 \\
\hline & & & 100 & 92.90 & 70.75 & 85.55 & 78.15 & 81.25 & 100.00 & 90.63 \\
\hline & & & 110 & 93.46 & 71.88 & 84.33 & 78.10 & 81.25 & 100.00 & 90.63 \\
\hline & & \multirow{2}{*}{ FC7 } & 60 & 94.78 & 67.14 & 82.42 & 74.78 & 81.25 & 100.00 & 90.63 \\
\hline & & & 70 & 95.48 & 69.92 & 86.04 & 77.98 & 81.25 & 100.00 & 90.63 \\
\hline & & \multirow{2}{*}{$\mathrm{FC} 8$} & 100 & 98.97 & 72.56 & 85.94 & 79.25 & 87.50 & 100.00 & 93.75 \\
\hline & & & 110 & 99.10 & 70.80 & 85.89 & 78.34 & 81.25 & 100.00 & 90.63 \\
\hline \multirow{5}{*}{ No } & \multirow{5}{*}{ Yes } & \multirow{2}{*}{ FC6 } & 100 & 92.53 & 67.14 & 71.19 & 69.17 & 75.00 & 87.50 & 81.25 \\
\hline & & & 110 & 93.13 & 68.31 & 71.44 & 69.87 & 75.00 & 87.50 & 81.25 \\
\hline & & \multirow{3}{*}{ FC7 } & 60 & 93.68 & 70.90 & 76.51 & 73.71 & 75.00 & 87.50 & 81.25 \\
\hline & & & 70 & 94.59 & 67.77 & 75.73 & 71.75 & 75.00 & 87.50 & 81.25 \\
\hline & & & 110 & 99.24 & 66.94 & 73.44 & 70.19 & 81.25 & 87.50 & 84.38 \\
\hline \multirow{6}{*}{ Yes } & \multirow{6}{*}{ Yes } & \multirow{2}{*}{ FC6 } & 60 & 85.81 & 84.03 & 89.26 & 86.65 & 93.75 & 100.00 & 96.88 \\
\hline & & & 70 & 87.29 & 79.88 & 87.26 & 83.57 & 93.75 & 100.00 & 96.88 \\
\hline & & \multirow{2}{*}{ FC7 } & 60 & 92.27 & 80.57 & 87.11 & 83.84 & 93.75 & 100.00 & 96.88 \\
\hline & & & 70 & 93.34 & 80.13 & 86.57 & 83.35 & 93.75 & 100.00 & 96.88 \\
\hline & & \multirow[b]{2}{*}{$\mathrm{FC} 8$} & 60 & 97.09 & 79.8 & 89.26 & 84.55 & 93.75 & 100.00 & 96.88 \\
\hline & & & 70 & 97.63 & 78.47 & 87.55 & 83.01 & 93.75 & 100.00 & 96.88 \\
\hline
\end{tabular}

\section{CONCLUSIONS}

This paper presents a complete framework for classification of SD-OCT images for DME detection. The methodology relies on preprocessing, deep feature extraction with AlexNet, data reduction through PCA while preserving at least $85 \%$ of the signal energy and classification based on linear SVMs. The results outperform previously published articles using the same data set. Future work will include a comparison with finetuning of deep architectures and fusion of different deep features as well as a more thorough evaluation of the effect of the volumes selected in the leave two out strategy.

\section{ACKNOWLEDGEMENTS}

The authors would like to thank Dr Carol Y. Cheung from HKCU and Tien Y. Wong from Singapore Eye Research Institute, for sharing the SERI dataset and FRGS grant FRGS/1/2017/TK04/UTP/01/1 Ministry of Higher Education (MOHE), Malaysia for supporting this research work.

\section{REFERENCES}

[1] J. Cunha-Vaz, "Diabetic macular edema," Eur J Ophthalmol, vol. 8, pp. 127-30, 1998.

[2] "Number of diabetics in Malaysia alarming," in The Star Online, ed. Kuala Lumpur: The Star, 2013, p. 1.

[3] K. M. Adal, D. Sidibé, S. Ali, E. Chaum, T. P. Karnowski, and F. Mériaudeau, "Automated detection of microaneurysms using scale-adapted blob analysis and semi-supervised learning," Computer methods and programs in biomedicine, vol. 114, pp. 1-10, 2014.

[4] S. Ali, D. Sidibé, K. M. Adal, L. Giancardo, E. Chaum, T. P. Karnowski, and F. Mériaudeau, "Statistical atlas based exudate segmentation," Computerized Medical Imaging and Graphics, vol. 37, pp. 358-368, 2013.

[5] R. Bernardes and J. Cunha-Vaz, Optical Coherence Tomography: A Clinical and Technical Update: Springer Berlin Heidelberg, 2012.

[6] M. Sonka and M. D. Abràmoff, "Quantitative analysis of retinal OCT," Medical Image Analysis, vol. 33, pp. 165169, 2016.

[7] G. Litjens, T. Kooi, B. E. Bejnordi, A. A. A. Setio, F. Ciompi, M. Ghafoorian J. A.van der Laak B. van 
Ginneken, and C. I. Sánchez, "A survey on deep learning in medical image analysis," arXiv preprint arXiv:1702.05747, 2017.

[8] N. Tajbakhsh, J. Y. Shin, S. R. Gurudu, R. T. Hurst, C. B. Kendall, M. B. Gotway, and J. Liang, "Convolutional Neural Networks for Medical Image Analysis: Full Training or Fine Tuning?," IEEE Transactions on Medical Imaging, vol. 35, pp. 1299-1312, 2016.

[9] P. Burlina, D. E. Freund, N. Joshi, Y. Wolfson, and N. M. Bressler, "Detection of age-related macular degeneration via deep learning," in Biomedical Imaging (ISBI), 2016 IEEE 13th International Symposium on, 2016, pp. 184- 188.

[10] S. P. K. Karri, D. Chakraborty, and J. Chatterjee, "Transfer learning based classification of optical coherence tomography images with diabetic macular edema and dry age-related macular degeneration," Biomedical Optics Express, vol. 8, pp. 579-592, 2017/02/01 2017.

[11] P. Sermanet, D. Eigen, X. Zhang, M. Mathieu, R. Fergus, and Y. LeCun, "Overfeat: Integrated recognition, localization and detection using convolutional networks," arXiv preprint arXiv:1312.6229, 2013.

[12] J. Massich, M. Rastgoo, G. Lemaitre, C. Cheung, T. Wong, D. Sidibé, and F. Mériaudeau, "Classifying DME vs Normal SD-OCT volumes: A review," in 23rd International Conference on Pattern Recognition, 2016.

[13] F. G. Venhuizen, B. van Ginneken, B. Bloemen, M. J. van Grinsven, R. Philipsen, C. Hoyng, T. Theelen, and C. I. Sánchez, "Automated age-related macular degeneration classification in OCT using unsupervised feature learning," in SPIE Medical Imaging, 2015, pp. 94141I-94141I-7.

[14] P. P. Srinivasan, L. A. Kim, P. S. Mettu, S. W. Cousins, G. M. Comer, J. A. Izatt, and S. Farsiu, "Fully automated detection of diabetic macular edema and dry age-related macular degeneration from optical coherence tomography images," Biomedical optics express, vol. 5, pp. 3568-3577, 2014.

[15] G. Lemaître, M. Rastgoo, J. Massich, S. Sankar, F. Mériaudeau, and D. Sidibé, "Classification of sd-oct volumes with lbp: Application to dme detection," in Proceedings of the Medical Image Computing and
Computer-Assisted Intervention (MICCA '15), Ophthalmic Medical Image Analysis Workshop (OMIA '15), Munich, Germany, 2015.

[16] K. Alsaih, G. Lemaître, J. M. Vall, M. Rastgoo, D. Sidibé, T. Y. Wong, E. Lamoureux, D. Milea, C. Y. Cheung, and F. Mériaudeau, "Classification of SD-OCT volumes with multi pyramids, LBP and HOG descriptors: application to DME detections," in Engineering in Medicine and Biology Society (EMBC), 2016 IEEE 38th Annual International Conference of the, 2016, pp. 1344-1347.

[17] Y. Y. Liu, M. Chen, H. Ishikawa, G. Wollstein, J. S. Schuman, and J. M. Rehg, "Automated macular pathology diagnosis in retinal OCT images using multi-scale spatial pyramid and local binary patterns in texture and shape encoding," Med Image Anal, vol. 15, 2011.

[18] S. Sankar, D. Sidibé, Y. Cheung, T. Wong, E. Lamoureux, D. Milea, and F. Mériaudeau, "Classification of sd-oct volumes for dme detection: an anomaly detection approach," in Medical Imaging 2016: Computer-Aided Diagnosis, 2016, p. 978520.

[19] G. C. Y. Chan, A. Muhammad, S. A. A. Shah, T. B. Tang, C.-K. Lu, and F. Meriaudeau, "Transfer Learning for Diabetic Macular Edema (DME) Detection on Optical Coherence Tomography (OCT) Images," in IEEE ICSIPA 2017, Kuching, Sarawak, Malaysia, 2017, pp. 1-4.

[20] A. Krizhevsky, I. Sutskever, and G. E. Hinton, "Imagenet classification with deep convolutional neural networks," in Advances in neural information processing systems, 2012, pp. 1097-1105.

[21] A. Muhammad, H. Muller, T. B. Tang, and F. Meriaudeau, "Classification of SD-OCT images using a Deep Learning Approach," in IEEE ICSIPA 2017, Kuching, Sarawak, Malaysia, 2017, pp. 1-4.

[22] I. T. Jolliffe, "Principal Component Analysis and Factor Analysis," in Principal component analysis, ed: Springer, 1986, pp. 115-128.

[23] G. Csurka, C. Dance, L. Fan, J. Willamowski, and C. Bray, "Visual categorization with bags of keypoints," in Workshop on statistical learning in computer vision, ECCV, 2004, pp. 1-2. 